\title{
Nationwide Sentinel Surveillance of Bloodstream Candida Infections in 40 Tertiary Care Hospitals in Spain $\nabla^{\nabla}$
}

\author{
R. Cisterna, ${ }^{1 *}$ G. Ezpeleta, ${ }^{1}$ O. Telleria,${ }^{1}$ J. Guinea ${ }^{2}$ B. Regueiro, ${ }^{3}$ J. Garcia-Rodríguez, ${ }^{4}$ \\ J. Esperalba, ${ }^{5}$ and The Spanish Candidemia Surveillance Group \\ Basurto Hospital, Clinical Microbiology and Infection Control Department, Bilbao, Spain ${ }^{1}$; Hospital Universitario Gregorio Marañón, \\ Clinical Microbiology Department, Madrid, Spain ${ }^{2}$; Complejo Hospitalario Universitario de Santiago, Clinical Microbiology Department, \\ Santiago de Compostela, Spain ${ }^{3}$; Hospital Universitario La Paz, Clinical Microbiology Department, Madrid, Spain ${ }^{4}$; \\ and Hospital Universitario Puerta de Hierro, Clinical Microbiology Department, Madrid, Spain ${ }^{5}$
}

Received 7 May 2010/Returned for modification 24 June 2010/Accepted 26 August 2010

\begin{abstract}
Candidemia studies have documented geographic differences in rates and epidemiology, underscoring the need for surveillance to monitor trends. We conducted prospective candidemia surveillance in Spain to assess the incidence, species distribution, frequency of antifungal resistance, and risk factors for acquiring a Candida infection. Prospective laboratory-based surveillance was conducted from June 2008 to June 2009 in 40 medical centers located around the country. A case of candidemia was defined as the isolation of a Candida species from a blood culture. Incidence rates were calculated per 1,000 admissions. Antifungal susceptibility tests were performed by using broth microdilution assay according to the guidelines of the Clinical and Laboratory Standards Institute. We detected 984 cases, for an overall incidence of 1.09 cases per 1,000 admissions. The crude mortality was $20.20 \%$. Candida albicans was the most common species $(\mathbf{4 9 . 0 8 \%})$, followed by $C$. parapsilosis $(\mathbf{2 0 . 7 3 \% ) , ~ C . ~ g l a b r a t a ~}(\mathbf{1 3 . 6 1 \%})$, and C. tropicalis $(\mathbf{1 0 . 7 7 \% )}$. Overall, decreased susceptibility to fluconazole occurred in $69(7.01 \%)$ incident isolates. Antifungal resistance was rare, and a moderate linear correlation between fluconazole and voriconazole MICs was observed. This is the largest multicenter candidemia study conducted to date and shows the substantial morbidity and mortality of candidemia in Spain.
\end{abstract}

Candida bloodstream infections (BSI) have become a major problem in tertiary care hospitals worldwide $(2,5,9,22,24,32$, 41). Candidemia is observed particularly among patients hospitalized for long periods who have been exposed to broadspectrum antimicrobial chemotherapy, immunosuppressive therapy, parenteral nutrition, and invasive medical procedures (7, 13). Despite some improvements in fungal BSI diagnosis during recent years, candidemia diagnosis remains difficult. In addition, candidemia is hard to treat and has a high attributable mortality rate, and its treatment is quite expensive $(16,27,45)$.

Although the incidence of candidemia among hospitalized patients increased during the 1980 s, more recent reports suggest that the incidence has stabilized. However, candidemia rates vary geographically. For example, an increasing incidence of candidemia in Iceland was reported for the period between 1980 and 1999 (5). On the other hand, data obtained from a national surveillance study conducted in Switzerland suggested that the incidence of candidemia remained unchanged during the period of 1991 to 2000 (24). It therefore seems that differences do exist in the epidemiology of candidemia between different countries, underscoring the need for continuous surveillance to monitor trends in incidence, species distribution, and antifungal drug susceptibility profiles.

\footnotetext{
* Corresponding author. Mailing address: Clinical Microbiology and Infection Control Department, Basurto Hospital, Avenida Montevideo 18, 48013 Bilbao, Spain. Phone: 34944006003 . Fax: 34944006301. E-mail: ramon.cisternacancer@osakidetza.net.

${ }^{\nabla}$ Published ahead of print on 8 September 2010.

$\dagger$ The authors have paid a fee to allow immediate free access to this article.
}

The epidemiology of candidemia has been studied extensively in the United States, Europe, and some countries in South America. Although there are some large studies published in this field, most of the data on candidemia in Spain are limited to retrospective reviews of medical records or observational studies conducted in a limited number of medical centers $(4,9,10,39)$. Consequently, the incidence of candidemia in tertiary care hospitals in Spain is largely unknown, and no reliable national data are available. We conducted a prospective laboratory-based surveillance study in 40 tertiary care hospitals representative of the Spanish public health system to assess the incidence, species distribution, frequency of antifungal resistance, and risk factors for candidemia.

\section{MATERIALS AND METHODS}

Surveillance. We performed prospective laboratory-based surveillance from June 2008 to June 2009 in 40 tertiary care academic medical centers located across the country. All hospitals had automated blood culture systems (Bactec or BacT-Alert) and provide medical care in different medical specialties to adults and children. The protocol was approved by the local institutional review board of each site.

Case definitions. A case of candidemia was defined as the incident isolation of a Candida species from a blood culture. An episode of candidemia occurring $>30$ days after the incident isolation was defined as a new case. Breakthrough candidemia was defined as the incident isolation of a Candida species from a blood culture from a patient receiving systemic antifungal therapy for any reason. Fever was defined as a body temperature of $\geq 37.8^{\circ} \mathrm{C}$, and neutropenia was defined as an absolute neutrophil count of $<500$ cells $/ \mathrm{mm}^{3}$. Patients were considered adults if their age was over 14 years.

Case accrual. In each center, when a candidemia case was identified, clinical and epidemiological data were collected prospectively with a standardized case report form. The case report form contained the following information: age, gender, date of admission, ward, date of candidemia, underlying medical conditions, exposure to invasive medical procedures, use of antibiotics or corticosteroids, management of candidemia, and outcome. The clinical case report list of 
TABLE 1. Demographics, clinical characteristics, and mortality for Candida BSI episodes identified during prospective sentinel surveillance conducted in Spain from June 2008 to June 2009

\begin{tabular}{|c|c|c|c|c|c|}
\hline \multirow{2}{*}{ Variable } & \multirow{2}{*}{$\begin{array}{l}\text { Value for all } \\
\text { cases }\end{array}$} & \multicolumn{4}{|c|}{ Value for cases with indicated species } \\
\hline & & C. albicans & C. parapsilosis & C. tropicalis & C. glabrata \\
\hline Median age (range) (yr) & $41(0-96)$ & $46(0-92)$ & $48(0-96)$ & $33(0-89)$ & $52(0-88)$ \\
\hline No. $(\%)$ of males & 577 (58.64) & $273(56.52)$ & $182(89.21)$ & $51(48.11)$ & $62(46.27)$ \\
\hline No. $(\%)$ of outpatients & $7(0.71)$ & $3(0.62)$ & $1(0.49)$ & $3(2.83)$ & $0(0.00)$ \\
\hline Median no. of days (range) in hospital until candidemia diagnosis & $20(0-385)$ & $20(0-114)$ & $19(0-385)$ & $19(0-47)$ & $19(0-115)$ \\
\hline \multicolumn{6}{|l|}{ No. $(\%)$ of cases of underlying disease } \\
\hline Cancer & $311(31.61)$ & $127(26.29)$ & $86(42.16)$ & $34(32.07)$ & $26(19.40)$ \\
\hline Hematological malignancy & $20(2.03)$ & $5(1.04)$ & $6(2.94)$ & $1(0.94)$ & $1(0.75)$ \\
\hline Coronary artery disease & $82(8.33)$ & $33(6.83)$ & $23(11.27)$ & $5(4.72)$ & $8(5.97)$ \\
\hline Chronic obstructive pulmonary disease & $71(7.21)$ & $40(8.28)$ & $11(5.39)$ & $5(4.72)$ & $9(6.71)$ \\
\hline Neurological disease & $35(3.55)$ & $14(2.80)$ & $12(5.88)$ & $2(1.89)$ & $2(1.49)$ \\
\hline Diabetes & $120(12.20)$ & $53(10.97)$ & $22(10.78)$ & $8(7.55)$ & $20(14.93)$ \\
\hline Organ transplantation & $45(4.57)$ & $14(2.90)$ & $21(10.29)$ & $2(1.89)$ & $3(2.23)$ \\
\hline HIV infection & $33(3.35)$ & $18(3.73)$ & $3(1.47)$ & $3(2.83)$ & $3(2.24)$ \\
\hline Parenteral drug abuse & $22(2.23)$ & $10(2.07)$ & $4(1.96)$ & $2(1.89)$ & $1(0.75)$ \\
\hline \multicolumn{6}{|l|}{ No. $(\%)$ of patients with characteristic } \\
\hline Previous or actual corticosteroid therapy & $180(18.29)$ & $80(16.56)$ & $50(24.50)$ & $13(12.26)$ & $21(15.67)$ \\
\hline Immunosuppressive therapy and/or neutropenia & $265(26.93)$ & $102(21.12)$ & 75 (36.76) & $28(26.41)$ & $28(20.90)$ \\
\hline In the ICU at diagnosis & $252(25.61)$ & $120(24.84)$ & $68(33.33)$ & $21(19.81)$ & $25(18.66)$ \\
\hline Mechanical ventilation & $265(26.93)$ & $133(27.54)$ & $71(34.80)$ & $15(14.15)$ & $26(19.40)$ \\
\hline On hemodialysis at diagnosis & $12(1.22)$ & $2(0.41)$ & $4(1.96)$ & $1(0.94)$ & $3(2.24)$ \\
\hline Previous surgery & $311(31.61)$ & $148(30.64)$ & $82(40.20)$ & $19(17.92)$ & $36(26.87)$ \\
\hline Central venous catheter & $659(66.79)$ & $295(61.07)$ & $187(91.66)$ & $52(49.06)$ & $61(45.52)$ \\
\hline Urinary catheter & $450(45.73)$ & $207(42.86)$ & $112(54.90)$ & $31(29.25)$ & $52(38.81)$ \\
\hline Prior antibiotic therapy & $747(75.91)$ & 337 (69.77) & $106(51.96)$ & $60(56.60)$ & $71(52.98)$ \\
\hline Prior fluconazole use & $78(7.93)$ & $29(6.00)$ & $10(4.90)$ & $12(11.32)$ & $29(21.32)$ \\
\hline Death attributed to candidemia & 134 (13.62) & $60(12.42)$ & $20(9.80)$ & $13(12.26)$ & $15(11.19)$ \\
\hline Mortality due to other conditions & 103 (10.47) & $48(9.94)$ & $36(17.65)$ & $7(6.60)$ & $14(10.45)$ \\
\hline Overall mortality & $237(24.10)$ & $108(22.36)$ & $56(27.45)$ & $20(18.87)$ & $29(21.64)$ \\
\hline
\end{tabular}

each hospital was compared with the isolates we received at Basurto Hospital for antifungal susceptibility testing in order to verify that neither cases nor isolates were missed. Moreover, audits of medical records were performed on $25 \%$ of cases to verify the accuracy and completeness of the data.

Yeast identification. All Candida species recovered from blood cultures were sent to the Mycology Laboratory of Basurto Hospital for confirmation of species identification and performance of antifungal agent susceptibility tests. Isolates were identified according to their morphology on Sabouraud chloramphenicol agar (BD, Franklin Lakes, NJ) and Chromagar Candida screening agar (BD, Franklin Lakes, NJ), by filamentation tests, and by biochemical tests in an API ID 32C system (bioMérieux AS, Marcy l'Etoile, France).

In vitro susceptibility testing. Antifungal susceptibility tests were performed by using broth microdilution assay according to the methodology recommended by CLSI (formerly known as NCCLS) document M27-A2 (28). The following antifungal drugs, supplied by the manufacturers as pure standard compounds, were tested at the indicated concentration range: amphotericin B, 0.015 to $8 \mu \mathrm{g} / \mathrm{ml}$ (Sigma Chemical Corporation, St. Louis, MO); caspofungin, 0.03 to $16 \mu \mathrm{g} / \mathrm{ml}$ (Merck \& Co., Inc., Whitehouse Station, NJ); fluconazole, 0.125 to $64 \mu \mathrm{g} / \mathrm{ml}$; voriconazole, 0.03 to $16 \mu \mathrm{g} / \mathrm{ml}$; and anidulafungin, 0.03 to $16 \mu \mathrm{g} / \mathrm{ml}$ (Pfizer Incorporated, New York, NY). Briefly, the medium used was RPMI 1640, with L-glutamine, without bicarbonate, and buffered at pH 7.0 with $0.165 \mathrm{M}$ morpholinepropanesulfonic acid (MOPS). The yeast inoculum suspension was prepared by using a spectrophotometer to obtain a final yeast concentration of $0.5 \times 10^{3}$ to $2.5 \times 10^{3}$ cells $/ \mathrm{ml}$ in each well of the microtiter plate. The assay mixtures were incubated at $35^{\circ} \mathrm{C}$ for $48 \mathrm{~h}$. Quality control strains (Candida parapsilosis ATCC 22019 and C. krusei ATCC 6258; American Type Culture Collection, Manassas, VA) were included on each day of assay to check the accuracy of the drug dilutions and the reproducibility of the results. The MIC endpoint for amphotericin B was considered the lowest tested drug concentration able to prevent any visible growth. The MIC for azoles and echinocandins was considered the lowest tested drug concentration causing a significant reduction (approximately 50\%) in growth compared to the growth of the drug-free positive control (28). The interpretative MIC breakpoints for azoles and echinocandins were those suggested in CLSI document M27-A2. Due to a lack of consensus about the definition of MIC breakpoints for amphotericin B, arbitrary values suggested from a previous study were used (29).

Statistical analysis. To calculate incidence rates, the numbers of admissions and patient-days were collected. Incidence rates were calculated as the number of candidemias per 1,000 admissions. The overall incidence was determined using summed denominators of patient-days and admissions to calculate pooled mean rates. In addition, during 12 months of surveillance, data on the different risk factors, underlying diseases, morbidity, and mortality were recorded. Data were recorded by using a case report form developed in Microsoft Access 2003 (Microsoft Corporation, Redmond, WA). Categorical data were analyzed using the chi-square test or Fisher's exact test, as appropriate, and continuous variables were compared using the Wilcoxon test. Spearman rank order correlation was used to measure the relationship between the MICs of fluconazole and voriconazole. We performed univariate and multivariate analyses of factors associated with candidemia caused by isolates with decreased susceptibility to fluconazole. Variables that had significant $P$ values $(<0.1)$ by univariate analysis were included in a multivariate model (backward and forward). Data were analyzed using SPSS 11.0.1 software (SPSS, Inc., Chicago, IL) and Stata (Stata Corporation, Lenexa, TX).

\section{RESULTS}

Demographics and incidence. We detected a total of 984 cases of candidemia during the surveillance study, for an overall incidence of 1.09 cases per 1,000 admissions. The incidence rates of candidemia in the 40 centers ranged from 0.76 to 1.49 cases per 1,000 admissions. Demographic and clinical characteristics and outcomes of 984 candidemia cases identified in the surveillance study are shown in Table 1 . Males comprised $64.02 \%$ of the case patients, and the median age was 41 years (range, 0 to 96 years). Notably, 189 case patients (15.2\%) were 
children, and $106(10.8 \%)$ were younger than 1 year old. The median age was 53 years among adult patients and 7 months among children.

Underlying diseases. At the time of diagnosis of candidemia, 975 case patients $(98.7 \%)$ were hospitalized, including 252 $(23.5 \%)$ admitted into the intensive care unit (ICU) (including the neonatal intensive care unit), $446(45.3 \%)$ in the medical ward, $173(17.6 \%)$ in the surgical ward, $73(7.41 \%)$ in the pediatric ward, and $40(4.06 \%)$ in other wards. Nine case patients were outpatients. Cancer was documented for 195 case patients $(19.84 \%)$, and 35 of these cancers $(17.94 \%)$ were hematologic malignancies (Table 1). A total of 311 case patients $(31.6 \%)$ had surgery in the 30 days before candidemia, and 199 of these surgeries $(64 \%)$ were abdominal. A total of 265 case patients $(26.93 \%)$ were on mechanical ventilation at the time of candidemia, $12(1.21 \%)$ were receiving dialysis, and $659(66.97 \%)$ had a central venous catheter. Neutropenia was present in only 35 case patients $(3.55 \%)$. Candidemia was generally a late complication during hospital stay, occurring a median of 19.5 days after admission (range, 0 to 385 days). Of the pediatric case patients, $36 \%$ had surgery in the 30 days prior to candidemia, $41 \%$ were in the ICU, and $25 \%$ were on mechanical ventilation.

Clinical manifestations of candidemia. Fever was the most frequent clinical manifestation of candidemia, occurring in 419 $(62 \%)$ of the 678 case patients for which data were available. Endocarditis was documented in only 17 cases (2\%), and Candida $\mathrm{sp}$. endophthalmitis was reported for 3 patients.

Therapy and outcome. At the time of candidemia, 122 case patients $(12.3 \%)$ were receiving a systemic antifungal agent and were considered to have breakthrough infections (78 patients were on fluconazole, 31 patients were on amphotericin B, 3 patients each were on itraconazole and voriconazole, and 5 patients were on echinocandins). Although the reason for this high rate of breakthrough infections is not clear, it is possible that other factors besides antifungal resistance have a role in this phenomenon. A deep analysis of these 122 cases showed that either the antifungal therapy duration or the election of the antifungal drug was inadequate. A total of 536 case patients $(54.5 \%)$ received antifungal therapy, started a median of 3 days from the isolation of Candida or the onset of candidemia (range, 0 to 27 days). The crude mortality rate was $24.10 \%$. The mortality rate among children was $16 \%$. Candidemia due to $C$. parapsilosis was associated with a lower mortality rate than that due to C. albicans $(9.80 \%$ versus $12.42 \%$; $P<0.02)$.

Species distribution and antifungal susceptibility testing. Table 2 shows the species distribution for the cases in this study. C. albicans was the most common species (483 cases [49.08\%]), followed by C. parapsilosis (204 cases [20.73\%]), C. glabrata (134 cases [13.61\%]), and C. tropicalis (106 cases [10.77\%]). C. krusei comprised only $5 \%$ of cases. Among adult patients, the distribution of Candida species was similar to that found for pediatric patients. Species distributions varied substantially between centers. The proportion of cases ranged between 27 and 54\% for C. albicans, between 16 and $29 \%$ for C. tropicalis, between 7 and $40 \%$ for C. parapsilosis, between 2 and $14 \%$ for C. glabrata, and between 0 and $9 \%$ for C. krusei (Table 2). Compared with candidemia due to C. albicans, candidemia due to $C$. tropicalis was less likely to occur among
TABLE 2. Species distribution and incidence among 984 cases of candidemia detected during prospective sentinel surveillance in Spain from June 2008 to June 2009

\begin{tabular}{lcc}
\hline \multicolumn{1}{c}{ Species } & No. (\%) of cases & $\begin{array}{c}\text { Range (\%) between } \\
\text { clinical settings }\end{array}$ \\
\hline C. albicans & $483(49.08)$ & $27-54$ \\
C. parapsilosis & $204(20.73)$ & $7-40$ \\
C. glabrata & $134(13.61)$ & $2-14$ \\
C. tropicalis & $106(10.77)$ & $16-29$ \\
C. krusei $^{a}$ & $21(2.13)$ & $0-9$ \\
Other species $^{a}$ & $36(3.65)$ & $0-4$ \\
\hline
\end{tabular}

${ }^{a}$ Species with fewer than 10 isolates recovered. This category includes $C$. famata, C. lusitaniae, C. pelliculosa, and Candida spp.

children of less than 1 year of age (12\% versus $22 \% ; P \leq 0.01)$ and more likely to occur in patients with cancer (32\% versus $26 \% ; P \leq 0.04)$ or neutropenia (8\% versus $3 \% ; P \leq 0.02)$. Compared with those due to $C$. albicans, cases due to $C$. parapsilosis were more likely among patients on mechanical ventilation $(35 \%$ versus $28 \% ; P \leq 0.03$ ) or receiving corticosteroids (24\% versus $16 \% ; P \leq 0.04)$. Most of these $C$. parapsilosis cases were in pediatric ICUs. Case patients receiving fluconazole before developing candidemia were more likely to be infected by $C$. glabrata than by $C$. albicans $(21 \%$ versus $6 \% ; P \leq 0.008)$ (Table 1). Table 3 shows the MIC ranges, $\mathrm{MIC}_{50} \mathrm{~s}, \mathrm{MIC}_{90} \mathrm{~s}$, and percentages of Candida isolates with decreased susceptibilities to the five antifungal drugs tested. Overall, decreased susceptibility to antifungal agents was rare. Five Candida albicans isolates exhibited a MIC of $\geq 4 \mu \mathrm{g} / \mathrm{ml}$ for voriconazole; these C. albicans isolates were also resistant to fluconazole. In addition, two C. krusei isolates that were resistant to fluconazole had a voriconazole MIC of $2 \mu \mathrm{g} / \mathrm{ml}$. Overall, for each isolate, there was a linear correlation between fluconazole and voriconazole MICs $(r=0.574 ; P \leq 0.01)$. In addition, patients who received fluconazole before candidemia had somewhat higher voriconazole MICs than those without previous exposure to fluconazole $\left(\mathrm{MIC}_{90} \mathrm{~s}\right.$ of $0.25 \mu \mathrm{g} / \mathrm{ml}$ and $0.06 \mu \mathrm{g} / \mathrm{ml}$, respectively; $P \leq 0.056$ ). In contrast to the case with susceptible isolates, candidemia caused by an isolate with decreased susceptibility (SDD) to fluconazole or resistant was associated with malignancy $(9 \%$ versus $3 \% ; P \leq 0.001)$, previous $(11 \%$ versus $4 \% ; P \leq 0.02$ ) or current (16\% versus $4 \% ; P \leq 0.001)$ neutropenia, and prior fluconazole use (14\% versus $3 \%$; $P \leq$ 0.001) (Table 4). By multivariate analysis, independent factors associated with candidemia due to an isolate with decreased susceptibility to fluconazole were neoplasia (odds ratio, 2.9; 95\% confidence interval, 1.4 to $5.9 ; P \leq 0.005$ ) and prior use of fluconazole (odds ratio, 3.8; 95\% confidence interval, 1.7 to 8.2; $P \leq 0.001)$. Among patients who received fluconazole as treatment for candidemia, the death rates for patients infected by susceptible isolates and less-susceptible isolates (SDD or resistant) were $54 \%$ and $64 \%$, respectively $(P \leq 0.44)$.

Caspofungin and anidulafungin resistance was low (16 cases for caspofungin and 8 cases for anidulafungin). Despite this low rate of in vitro resistance to echinocandins for the isolates studied, MICs for C. parapsilosis and C. guilliermondii were higher than the MICs obtained for other Candida spp., as described in other studies across North America and Europe. 
TABLE 3. Antifungal susceptibility test results for selected species of Candida isolated during prospective sentinel surveillance in Spain from June 2008 to June 2009

\begin{tabular}{|c|c|c|c|c|c|}
\hline Species $(n)$ & Antifungal agent & $\begin{array}{l}\text { MIC range } \\
(\mu \mathrm{g} / \mathrm{ml})\end{array}$ & $\begin{array}{l}\mathrm{MIC}_{50} \\
(\mu \mathrm{g} / \mathrm{ml})\end{array}$ & $\begin{array}{l}\mathrm{MIC}_{90} \\
(\mu \mathrm{g} / \mathrm{ml})\end{array}$ & $\begin{array}{l}\text { No. }(\%) \text { of } \\
\text { resistant or } \\
\text { SDD isolates }\end{array}$ \\
\hline \multirow[t]{5}{*}{ C. albicans (483) } & Amphotericin B & $0.125-1.0$ & 0.5 & 1.0 & $0(0.00)$ \\
\hline & Fluconazole & $0.125-64$ & 0.5 & 2.0 & $10(2.07)$ \\
\hline & Voriconazole & $<0.03-4$ & 0.03 & 0.03 & $5(1.04)$ \\
\hline & Caspofungin & $<0.03-4$ & 0.25 & 2.0 & $3(0.62)$ \\
\hline & Anidulafungin & $<0.03-2$ & 0.03 & 0.03 & $0(0.00)$ \\
\hline \multirow[t]{5}{*}{ C. parapsilosis (204) } & Amphotericin B & $0.25-1.0$ & 1.0 & 1.0 & $0(0.00)$ \\
\hline & Fluconazole & $0.125-64$ & 0.5 & 4.0 & $11(5.39)^{a}$ \\
\hline & Voriconazole & $0.03-2$ & 0.03 & 0.125 & $3(1.47)^{b}$ \\
\hline & Caspofungin & $0.125-64$ & 1.0 & 2.0 & $12(5.88)$ \\
\hline & Anidulafungin & $0.03-0.25$ & 1.0 & 2.0 & $7(3.43)$ \\
\hline \multirow[t]{5}{*}{ C. glabrata (134) } & Amphotericin B & $0.25-1.0$ & 0.5 & 1.0 & $0(0.00)$ \\
\hline & Fluconazole & 1.0-64 & 4.0 & 32 & 25 (18.67) \\
\hline & Voriconazole & $0.03-4.0$ & 0.125 & 0.5 & $3(2.24)$ \\
\hline & Caspofungin & $0.03-0.5$ & 0.06 & 0.125 & $0(0.00)$ \\
\hline & Anidulafungin & $0.06-0.5$ & 0.06 & 0.125 & $0(0.00)$ \\
\hline \multirow[t]{5}{*}{ C. tropicalis (106) } & Amphotericin B & $0.125-1.0$ & 0.5 & 1.0 & $0(0.00)$ \\
\hline & Fluconazole & $0.25-128$ & 1 & 2 & $2(1.89)$ \\
\hline & Voriconazole & $0.03-8$ & 0.06 & 0.125 & $2(1.89)$ \\
\hline & Caspofungin & $0.03-0.5$ & 0.06 & 0.125 & $0(0.00)$ \\
\hline & Anidulafungin & $0.03-0.5$ & 0.03 & 0.125 & $0(0.00)$ \\
\hline \multirow[t]{5}{*}{ C. krusei (21) } & Amphotericin B & $0.125-2.0$ & 0.25 & 0.75 & $0(0.00)$ \\
\hline & Fluconazole & 16-128 & 4.0 & 8.0 & $21(100.00)$ \\
\hline & Voriconazole & $0.03-4.0$ & 0.25 & 1.0 & $2(9.52)$ \\
\hline & Caspofungin & $0.125-8$ & 0.125 & 1.0 & $1(4.76)$ \\
\hline & Anidulafungin & $0.03-8$ & 0.06 & 0.25 & $1(4.76)$ \\
\hline
\end{tabular}

${ }^{a}$ All but one of the isolates exhibited decreased susceptibility (SDD) to fluconazole.

${ }^{b}$ All of the isolates exhibited decreased susceptibility to voriconazole.

\section{DISCUSSION}

This prospective candidemia surveillance study represents one of the largest multicenter studies conducted in Spain and reveals for the first time the large burden of candidemia in Spanish tertiary care hospitals. In addition, it provides the most representative and consistent data from Spain on the epidemiology of candidemia to date. The first remarkable finding of our study was the incidence of candidemia. Our rates are higher than those reported for centers in the Northern Hemisphere, including the United States $(0.28$ to 0.96 case per 1,000 admissions $)(6,34,35,43)$, Canada ( 0.45 case per 1,000 admissions) (23), Europe ( 0.20 to 0.38 case per 1,000 admissions) (41), France (0.17 case per 1,000 admissions) (37), Norway (0.17 case per 1,000 admissions) (38), Hungary (0.20 to 0.40

TABLE 4. Summary of univariate statistical analysis between fluconazole-susceptible isolates and resistant or SDD ones ${ }^{a}$

\begin{tabular}{lccl}
\hline \multicolumn{1}{c}{ Variable } & $\begin{array}{c}\text { No. (\%) of isolates } \\
\text { susceptible to } \\
\text { fluconazole }\end{array}$ & $\begin{array}{c}\text { No. (\%) of isolates } \\
\text { with decreased } \\
\text { susceptibility or } \\
\text { with resistance to } \\
\text { fluconazole }\end{array}$ & $P$ value \\
\hline Neoplasia & $27(2.95)$ & $6(8.70)$ & $\leq 0.001$ \\
Neutropenia & $37(4.04)$ & $11(15.94)$ & $\leq 0.001$ \\
Prior fluconazole use & $27(2.95)$ & $10(14.49)$ & $\leq 0.001$ \\
\hline
\end{tabular}

${ }^{a}$ Only statistically significant variables are summarized in the table. case per 1,000 admissions) (12), Switzerland (0.27 case per 1,000 admissions) $(24,36)$, Italy $(0.38$ case per 1,000 admissions) (40), and Spain ( 0.76 to 0.81 case per 1,000 admissions) $(2,3,42,44)$. Although the reasons for our high candidemia rates are not entirely clear, it is possible that they may be related to a combination of multiple factors, including differences in resources available for medical care and transplantation programs, difficulties in the implementation of infection control programs in hospitals, limited numbers of health care workers to assist patients in critical care units, and less-aggressive practices of empirical antifungal therapy and prophylaxis for high-risk patients. Another possibility is that most of the revised data on incidence rates are from the late 1980s and late 1990s and may not reflect the current trends. Nevertheless, since our rates of BSI caused by other organisms are also high, it seems that these factors may have affected not just candidemia rates but also the overall rates of BSI in Spain. The median age of our case patients was low (41 years) compared to that reported in a series from the United States (32). This is a reflection of the large proportion of children in our study, especially in the Spanish hospitals located in the southern part of the country (32\% compared to $9 \%$ in the study from the United States). The median age of our adults (57 years) was similar to that reported in the American study (55 years). Most of the children were premature babies (40\%).

The adults with candidemia comprised mostly case patients admitted to an ICU, with diseases such as diabetes, cardiac 
diseases, and lung disease. Nearly half of the ICU case patients had surgery in the 30 days before candidemia, underscoring the importance of surgical procedures and location in the ICU in the epidemiology of candidemia (38). Over the past 10 years, some studies have reported a shift in the etiology of candidemia. While $C$. albicans is still considered the most common species causing candidemia, increasing rates of candidemia caused by $C$. tropicalis, $C$. parapsilosis, C. glabrata, and C. krusei have been reported worldwide $(9,37,42)$. The reasons for the emergence of non-C. albicans species are not completely understood, but some medical conditions may consistently impact the risk of developing candidemia due to non-C. albicans species: $C$. parapsilosis fungemia has been associated with vascular catheters and parenteral nutrition $(8,15,20), C$. tropicalis candidemia is associated with cancer and neutropenia (19), and $C$. krusei and $C$. glabrata fungemias are associated with previous exposure to azoles $(21,25,42)$. The findings from our surveillance are supportive of these reports. Our series clearly consolidates the concept that candidemia due to $C$. krusei is rare in Spain and shows that $C$. parapsilosis accounts for the large majority of non-C. albicans species. Why $C$. krusei is unusual in Spain is not clear, but the wide geographic variability in the species distribution suggests that factors other than the use of fluconazole may be important, including demographic characteristics and the use of antibiotics. However, although the proportion of $C$. krusei infections in Spain is small, the burden of $C$. krusei candidemia is more or less similar to rates reported in other series (3). We report a low rate of $C$. tropicalis candidemia, even in neonates, similar to those published in European and North American series (2 to $10 \%$ in Europe and 10 to $12 \%$ in the United States and Canada) $(2,3,5,11,14,23,25$, $32,41,42)$, whereas a larger proportion was observed in a study from Saudi Arabia (20.7\%) (1). C. parapsilosis candidemia was frequent in the present study. There is wide variation in the proportion of cases with $C$. parapsilosis as the cause of candidemia in different series, with rates varying between 7 and $21 \%$ in the United States $(11,18)$ and between 6.9 and $30 \%$ in Europe $(41,42)$. The reasons for these wide variations are not clear but may be related to the proportion of neonates in each study as well as to other local epidemiologic factors for this species, since $C$. parapsilosis candidemia is thought to be acquired from an external source. We also observed two cases of candidemia caused by C. pelliculosa, the asexual form of Pichia anomala. C. pelliculosa has rarely been reported as a cause of candidemia outside outbreaks, two of which occurred in South America (33). Both cases of $C$. pelliculosa isolates were from the same region and suggest the possibility that this species may be associated with nosocomial outbreaks or a particular geographical distribution. However, further investigation is needed to understand the epidemiology of and factors associated with $C$. pelliculosa infection. Antifungal resistance was a rare finding in our study and was restricted to a few isolates. As with two recently published studies $(2,31)$, none of our Candida bloodstream isolates had MICs of $>2 \mu \mathrm{g} / \mathrm{ml}$ for amphotericin B. Our proportion of fluconazole-resistant isolates $(6.32 \%)$ was similar to the rates observed with European $(5.2 \%)$ and North American (6.6\%) isolates (26). As reported by other authors (25), we also observed that previous exposure to fluconazole was a strong and independent factor associated with candidemia caused by fluconazole-nonsusceptible iso- lates. In addition, we observed that higher voriconazole MICs tended to be associated with prior exposure to fluconazole. This is of concern and illustrates the potential problem of cross-resistance between azoles. In another study with 46 fluconazole-resistant C. glabrata isolates, only $13 \%$ were susceptible to voriconazole (33). The potential for voriconazole-resistant $C$. glabrata to emerge as a threat in people receiving voriconazole therapy has been raised in two reports of breakthrough infections $(1,17)$. Nevertheless, in our study, voriconazole was the azole which exhibited the best in vitro antifungal activity, and only one of six fluconazole-resistant isolates was cross-resistant to voriconazole.

The crude mortality rate observed in our study was similar to those reported by other authors $(2,9,16)$. Adults had higher mortality rates than children between 1 and 14 years of age ( $24.10 \%$ and $16 \%$, respectively). Similar to other reports, patients with $C$. parapsilosis candidemia had the lowest death rates $(30,32)$. These comprehensive data document important differences in the epidemiology of candidemia in Spain and reports from other countries. This report shows that candidemia is a significant source of morbidity in Spain, with a substantial burden of disease, mortality, and likely high associated costs. Although our high rates of candidemia may be related to high rates of BSI in general in Spanish public hospitals, reasons for these high rates are not clear and warrant further study. Determining factors associated with these high rates may lead to identifying measures that can help to prevent disease. In addition, our data support the observation that fluconazole nonsusceptibility is associated with prior fluconazole exposure and suggest that such exposure may lead to voriconazole cross-resistance.

\section{ACKNOWLEDGMENTS}

This paper was partially supported by an independent medical grant provided by Pfizer, Inc., and The Basque Foundation for Health Innovation and Research (BIOEF).

We express our gratitude to all the persons involved in The Spanish Candidemia Surveillance Group, whose composition is detailed below.

The Spanish Candidemia Surveillance Group is composed of the following persons and hospitals in Spain: Complejo Hospitalario de Ourense (B. Fernández); Complejo Hospitalario J. Canalejo de Coruña (D. Velasco); Complejo Hospitalario de Santiago (B. Regueiro); Complexo Hospitalario Xeral Calde, Lugo (F. García Garrote); Hospital Arquitecto Marcide, Ferrol (P. Ordóñez); Complejo Hospitalario Xeral Cies, Pontevedra (I. Iglesias); Hospital de Leon (M. I. Fernández-Natal); Hospital Clínico de Salamanca (M. I. García); Hospital Clínico de Valladolid (R. Ortiz de Lejarazu and C. Gobernado); Hospital Clínico de Badajoz (J. Blanco); Hospital San Pedro de Alcántara, Cáceres (P. Hernández); Hospital Virgen del Rocio, Sevilla (J. Aznar); Hospital Reina Sofía, Córdoba (M. Casal); Hospital de Valme, Sevilla (E. Martín); Hospital Puerta del Mar, Cádiz (A. M. García-Tapia and C. Fernandez); Hospital Virgen de la Nieves, Granada (A. Sampedro); Hospital de Canarias, Tenerife (S. Campos); Hospital La Paz, Madrid (J. García-Rodríguez); Hospital Puerta de Hierro, Madrid (J. Esperalba); Hospital Gregorio Marañón, Madrid (J. Guinea); Hospital Clínico San Carlos, Madrid (P. Merino); Hospital General de Castellón (B. Gomila); Hospital La Fe, Valencia (M. Gobernado and J. Pemán); Hospital Dr. Peset, Valencia (J. J. Camarena); Hospital de Alicante (J. Plazas); Hospital General de Elche, Alicante (G. Royo); Hospital J. M. Morales Messeguer, Murcia (R. M. Blázquez); Hospital Virgen de la Arrixaca, Murcia (G. Yagüe and M. A. Iborra); Complejo Hospitalario de Albacete (J. J. Palomar); Hospital Son Dureta, Palma de Mallorca (J. L. Pérez); Hospital Son Llatzer, Baleares (M. M. Garau); Hospital Vall d'Hebron, Barcelona (G. Prats and V. Rodríguez); Hospital Clinic, Barcelona (T. Jiménez de Anta and C. 
Pitart); Hospital Universitari Bellvitge, Barcelona (J. Ayats); Hospital Miguel Servet, Zaragoza (M. J. Revillo and A. Rezusta); Hospital Virgen del Camino, Pamplona (L. Torroba-Álvarez); Hospital Donosita, Guipúzcoa (M. J. Echeverría); Hospital Marqués de Valdecilla, Santander (M. Gozalo); and Hospital de Basurto, Bilbao (R. Cisterna, G. Ezpeleta, and O. Telleria).

\section{REFERENCES}

1. Al-Jasser, A. M., and N. A. Elkhizzi. 2004. Distribution of Candida species among bloodstream isolates. Saudi Med. J. 25:566-569.

2. Almirante, B., D. Rodriguez, B. J. Park, M. Cuenca-Estrella, A. M. Planes, M. Almela, J. Mensa, F. Sanchez, J. Ayats, M. Gimenez, P. Saballs, S. K. Fridkin, J. Morgan, J. L. Rodriguez-Tudela, D. W. Warnock, and A. Pahissa 2005. Epidemiology and predictors of mortality in cases of Candida bloodstream infection: results from population-based surveillance, Barcelona Spain, from 2002 to 2003. J. Clin. Microbiol. 43:1829-1835.

3. Alonso-Valle, H., O. Acha, J. D. Garcia-Palomo, C. Farinas-Alvarez, C Fernandez-Mazarrasa, and M. C. Farinas. 2003. Candidemia in a tertiary care hospital: epidemiology and factors influencing mortality. Eur. J. Clin. Microbiol. Infect. Dis. 22:254-257.

4. Antunes, A. G., A. C. Pasqualotto, M. C. Diaz, P. A. d'Azevedo, and L. C. Severo. 2004. Candidemia in a Brazilian tertiary care hospital: species distribution and antifungal susceptibility patterns. Rev. Inst. Med. Trop. Sao Paulo 46:239-241.

5. Asmundsdottir, L. R., H. Erlendsdottir, and M. Gottfredsson. 2002. Increasing incidence of candidemia: results from a 20-year nationwide study in Iceland. J. Clin. Microbiol. 40:3489-3492.

6. Banerjee, S. N., T. G. Emori, D. H. Culver, R. P. Gaynes, W. R. Jarvis, T. Horan, J. R. Edwards, J. Tolson, T. Henderson, W. J. Martone, et al. 1991 Secular trends in nosocomial primary bloodstream infections in the United States, 1980-1989. Am. J. Med. 91:86S-89S

7. Blumberg, H. M., W. R. Jarvis, J. M. Soucie, J. E. Edwards, J. E. Patterson, M. A. Pfaller, M. S. Rangel-Frausto, M. G. Rinaldi, L. Saiman, R. T. Wiblin R. P. Wenzel, et al. 2001. Risk factors for candidal bloodstream infections in surgical intensive care unit patients: the NEMIS prospective multicenter study. Clin. Infect. Dis. 33:177-186.

8. Clark, T. A., S. A. Slavinski, J. Morgan, T. Lott, B. A. Arthington-Skaggs, M. E. Brandt, R. M. Webb, M. Currier, R. H. Flowers, S. K. Fridkin, and R. A. Hajjeh. 2004. Epidemiologic and molecular characterization of an outbreak of Candida parapsilosis bloodstream infections in a community hospital. J. Clin. Microbiol. 42:4468-4472.

9. Colombo, A. L., M. Nucci, R. Salomao, M. L. Branchini, R. Richtmann, A. Derossi, and S. B. Wey. 1999. High rate of non-albicans candidemia in Brazilian tertiary care hospitals. Diagn. Microbiol. Infect. Dis. 34:281-286.

10. Costa, S. F., I. Marinho, E. A. Araujo, A. E. Manrique, E. A. Medeiros, and A. S. Levin. 2000. Nosocomial fungaemia: a 2-year prospective study. J. Hosp. Infect. 45:69-72.

11. Diekema, D. J., S. A. Messer, A. B. Brueggemann, S. L. Coffman, G. V Doern, L. A. Herwaldt, and M. A. Pfaller. 2002. Epidemiology of candidemia: 3-year results from the emerging infections and the epidemiology of Iowa organisms study. J. Clin. Microbiol. 40:1298-1302.

12. Doczi, I., E. Dosa, E. Hajdu, and E. Nagy. 2002. Aetiology and antifungal susceptibility of yeast bloodstream infections in a Hungarian university hospital between 1996 and 2000. J. Med. Microbiol. 51:677-681.

13. Fraser, V. J., M. Jones, J. Dunkel, S. Storfer, G. Medoff, and W. C. Dunagan. 1992. Candidemia in a tertiary care hospital: epidemiology, risk factors, and predictors of mortality. Clin. Infect. Dis. 15:414-421.

14. Garbino, J., L. Kolarova, P. Rohner, D. Lew, P. Pichna, and D. Pittet. 2002. Secular trends of candidemia over 12 years in adult patients at a tertiary care hospital. Medicine (Baltimore) 81:425-433.

15. Girmenia, C., P. Martino, F. De Bernardis, G. Gentile, M. Boccanera, M Monaco, G. Antonucci, and A. Cassone. 1996. Rising incidence of Candida parapsilosis fungemia in patients with hematologic malignancies: clinical aspects, predisposing factors, and differential pathogenicity of the causative strains. Clin. Infect. Dis. 23:506-514.

16. Gudlaugsson, O., S. Gillespie, K. Lee, B. J. Vande, J. Hu, S. Messer, L. Herwaldt, M. Pfaller, and D. Diekema. 2003. Attributable mortality of nosocomial candidemia, revisited. Clin. Infect. Dis. 37:1172-1177.

17. Imhof, A., S. A. Balajee, D. N. Fredricks, J. A. Englund, and K. A. Marr 2004. Breakthrough fungal infections in stem cell transplant recipients receiving voriconazole. Clin. Infect. Dis. 39:743-746.

18. Kao, A. S., M. E. Brandt, W. R. Pruitt, L. A. Conn, B. A. Perkins, D. S Stephens, W. S. Baughman, A. L. Reingold, G. A. Rothrock, M. A. Pfaller, R. W. Pinner, and R. A. Hajjeh. 1999. The epidemiology of candidemia in two United States cities: results of a population-based active surveillance. Clin. Infect. Dis. 29:1164-1170.

19. Komshian, S. V., A. K. Uwaydah, J. D. Sobel, and L. R. Crane. 1989 Fungemia caused by Candida species and Torulopsis glabrata in the hospitalized patient: frequency, characteristics, and evaluation of factors influencing outcome. Rev. Infect. Dis. 11:379-390.

20. Levy, I., L. G. Rubin, S. Vasishtha, V. Tucci, and S. K. Sood. 1998. Emer- gence of Candida parapsilosis as the predominant species causing candidemia in children. Clin. Infect. Dis. 26:1086-1088.

21. Lin, M. Y., Y. Carmeli, J. Zumsteg, E. L. Flores, J. Tolentino, P. Sreeramoju, and S. G. Weber. 2005. Prior antimicrobial therapy and risk for hospital acquired Candida glabrata and Candida krusei fungemia: a case-case-contro study. Antimicrob. Agents Chemother. 49:4555-4560.

22. Luzzati, R., G. Amalfitano, L. Lazzarini, F. Soldani, S. Bellino, M. Solbiati, M. C. Danzi, S. Vento, G. Todeschini, C. Vivenza, and E. Concia. 2000 Nosocomial candidemia in non-neutropenic patients at an Italian tertiary care hospital. Eur. J. Clin. Microbiol. Infect. Dis. 19:602-607.

23. Macphail, G. L., G. D. Taylor, M. Buchanan-Chell, C. Ross, S. Wilson, and A. Kureishi. 2002. Epidemiology, treatment and outcome of candidemia: a five-year review at three Canadian hospitals. Mycoses 45:141-145.

24. Marchetti, O., J. Bille, U. Fluckiger, P. Eggimann, C. Ruef, J. Garbino, T. Calandra, M. P. Glauser, M. G. Tauber, and D. Pittet. 2004. Epidemiology of candidemia in Swiss tertiary care hospitals: secular trends, 1991-2000. Clin. Infect. Dis. 38:311-320.

25. Marr, K. A., K. Seidel, T. C. White, and R. A. Bowden. 2000. Candidemia in allogeneic blood and marrow transplant recipients: evolution of risk factors after the adoption of prophylactic fluconazole. J. Infect. Dis. 181:309-316.

26. Messer, S. A., G. J. Moet, J. T. Kirvy, and R. N. Jones. 2009. Activity of contemporary antifungal agents, including the novel echinocandin anidulafungin, tested against Candida spp., Cryptococcus spp., and Aspergillus spp.: report from the SENTRY antimicrobial surveillance program (2006 to 2007). J. Clin. Microbiol. 47:1942-1946.

27. Morgan, J., M. I. Meltzer, B. D. Plikaytis, A. N. Sofair, S. Huie-White, S. Wilcox, L. H. Harrison, E. C. Seaberg, R. A. Hajjeh, and S. M. Teutsch. 2005 Excess mortality, hospital stay, and cost due to candidemia: a case-control study using data from population-based candidemia surveillance. Infect. Control Hosp. Epidemiol. 26:540-547.

28. National Committee for Clinical Laboratory Standards. 2002. Reference method for broth dilution antifungal susceptibility testing of yeasts; approved standard, 2nd ed. Document M27-A2. National Committee for Clinical Laboratory Standards, Wayne, PA.

29. Nguyen, M. H., C. J. Clancy, V. L. Yu, Y. C. Yu, A. J. Morris, D. R. Snydman, D. A. Sutton, and M. G. Rinaldi. 1998. Do in vitro susceptibility data predict the microbiologic response to amphotericin B? Results of a prospective study of patients with Candida fungemia. J. Infect. Dis. 177:425-430.

30. Nucci, M., A. L. Colombo, F. Silveira, R. Richtmann, R. Salomao, M. L. Branchini, and N. Spector. 1998. Risk factors for death in patients with candidemia. Infect. Control Hosp. Epidemiol. 19:846-850.

31. Ostrosky-Zeichner, L., J. H. Rex, P. G. Pappas, R. J. Hamill, R. A. Larsen, H. W. Horowitz, W. G. Powderly, N. Hyslop, C. A. Kauffman, J. Cleary, J. E. Mangino, and J. Lee. 2003. Antifungal susceptibility survey of 2,000 bloodstream Candida isolates in the United States. Antimicrob. Agents Chemother. 47:3149-3154.

32. Pappas, P. G., J. H. Rex, J. Lee, R. J. Hamill, R. A. Larsen, W. Powderly, C. A. Kauffman, N. Hyslop, J. E. Mangino, S. Chapman, H. W. Horowitz, J. E. Edwards, and W. E. Dismukes. 2003. A prospective observational study of candidemia: epidemiology, therapy, and influences on mortality in hospitalized adult and pediatric patients. Clin. Infect. Dis. 37:634-643.

33. Pasqualotto, A. C., T. C. Sukiennik, L. C. Severo, C. S. de Amorim, and A. L. Colombo. 2005. An outbreak of Pichia anomala fungemia in a Brazilian pediatric intensive care unit. Infect. Control Hosp. Epidemiol. 26:553-558.

34. Pfaller, M. A., R. N. Jones, G. V. Doern, H. S. Sader, R. J. Hollis, and S. A. Messer for the SENTRY Participant Group. 1998. International surveillance of bloodstream infections due to Candida species: frequency of occurrence and antifungal susceptibilities of isolates collected in 1997 in the United States, Canada, and South America for the SENTRY program. J. Clin. Microbiol. 36:1886-1889.

35. Pfaller, M. A., S. A. Messer, L. Boyken, S. Tendolkar, R. J. Hollis, and D. J. Diekema. 2004. Geographic variation in the susceptibilities of invasive isolates of Candida glabrata to seven systemically active antifungal agents: a global assessment from the ARTEMIS Antifungal Surveillance Program conducted in 2001 and 2002. J. Clin. Microbiol. 42:3142-3146.

36. Pittet, D., and R. P. Wenzel. 1995. Nosocomial bloodstream infections. Secular trends in rates, mortality, and contribution to total hospital deaths. Arch. Intern. Med. 155:1177-1184.

37. Richet, H., P. Roux, C. C. Des, Y. Esnault, and A. Andremont. 2002. Candidemia in French hospitals: incidence rates and characteristics. Clin. Microbiol. Infect. 8:405-412.

38. Sandven, P., L. Bevanger, A. Digranes, P. Gaustad, H. H. Haukland, and M. Steinbakk. 1998. Constant low rate of fungemia in Norway, 1991 to 1996. The Norwegian Yeast Study Group. J. Clin. Microbiol. 36:3455-3459.

39. San Miguel, L. G., J. Cobo, E. Otheo, A. Sanchez-Sousa, V. Abraira, and S. Moreno. 2005. Secular trends of candidemia in a large tertiary-care hospital from 1988 to 2000: emergence of Candida parapsilosis. Infect. Control Hosp. Epidemiol. 26:548-552.

40. Silva, V., M. C. Diaz, and N. Febre. 2004. Invasive fungal infections in Chile: a multicenter study of fungal prevalence and susceptibility during a 1-year period. Med. Mycol. 42:333-339. 
41. Tortorano, A. M., E. Biraghi, A. Astolfi, C. Ossi, M. Tejada, C. Farina, S. Perin, C. Bonaccorso, C. Cavanna, A. Raballo, and A. Grossi. 2002 European Confederation of Medical Mycology (ECMM) prospective survey of candidaemia: report from one Italian region. J. Hosp. Infect. 51:297-304.

42. Tortorano, A. M., J. Peman, H. Bernhardt, L. Klingspor, C. C. Kibbler, O. Faure, E. Biraghi, E. Canton, K. Zimmermann, S. Seaton, and R. Grillot. 2004. Epidemiology of candidaemia in Europe: results of 28-month European Confederation of Medical Mycology (ECMM) hospital-based surveillance study. Eur. J. Clin. Microbiol. Infect. Dis. 23:317-322.
43. Trick, W. E., S. K. Fridkin, J. R. Edwards, R. A. Hajjeh, and R. P. Gaynes. 2002. Secular trend of hospital-acquired candidemia among intensive care unit patients in the United States during 1989-1999. Clin. Infect. Dis. 35:627-630.

44. Viudes, A., J. Peman, E. Canton, P. Ubeda, J. L. Lopez-Ribot, and M. Gobernado. 2002. Candidemia at a tertiary-care hospital: epidemiology, treatment, clinical outcome and risk factors for death. Eur. J. Clin. Microbiol. Infect. Dis. 21:767-774.

45. Weems, J. J., Jr. 1992. Candida parapsilosis: epidemiology, pathogenicity, clinical manifestations, and antimicrobial susceptibility. Clin. Infect. Dis. 14:756-766 